\title{
Diagnosis of Fanconi Anemia: Mutation Analysis by Multiplex Ligation-Dependent Probe Amplification and PCR-Based Sanger Sequencing
}

\author{
Johan J. P. Gille, Karijn Floor, Lianne Kerkhoven, Najim Ameziane, \\ Hans Joenje, and Johan P. de Winter \\ Department of Clinical Genetics, VU University Medical Center, Van der Boechorsttraat 7, 1081 BT Amsterdam, The Netherlands \\ Correspondence should be addressed to Johan J. P. Gille, jjp.gille@vumc.nl
}

Received 22 December 2011; Accepted 21 March 2012

Academic Editor: Stefan Meyer

Copyright (c) 2012 Johan J. P. Gille et al. This is an open access article distributed under the Creative Commons Attribution License, which permits unrestricted use, distribution, and reproduction in any medium, provided the original work is properly cited.

\begin{abstract}
Fanconi anemia (FA) is a rare inherited disease characterized by developmental defects, short stature, bone marrow failure, and a high risk of malignancies. FA is heterogeneous: 15 genetic subtypes have been distinguished so far. A clinical diagnosis of FA needs to be confirmed by testing cells for sensitivity to cross-linking agents in a chromosomal breakage test. As a second step, DNA testing can be employed to elucidate the genetic subtype of the patient and to identify the familial mutations. This knowledge allows preimplantation genetic diagnosis (PGD) and enables prenatal DNA testing in future pregnancies. Although simultaneous testing of all FA genes by next generation sequencing will be possible in the near future, this technique will not be available immediately for all laboratories. In addition, in populations with strong founder mutations, a limited test using Sanger sequencing and MLPA will be a cost-effective alternative. We describe a strategy and optimized conditions for the screening of FANCA, FANCB, FANCC, FANCE, FANCF, and FANCG and present the results obtained in a cohort of 54 patients referred to our diagnostic service since 2008. In addition, the follow up with respect to genetic counseling and carrier screening in the families is discussed.
\end{abstract}

\section{Introduction}

Fanconi anemia (FA) is a rare inherited syndrome with diverse clinical symptoms including developmental defects, short stature, bone marrow failure, and a high risk of malignancies. Fifteen genetic subtypes have been distinguished: FA-A, -B, -C, -D1, -D2, -E, -F, -G, -I, -J, - L, -M, -N, -O, and -P. [1-4]. The majority of patients $(\sim 85 \%)$ belong to the subtypes A $(\sim 60 \%), \mathrm{C}(\sim 10-15 \%)$, or G $(\sim 10 \%)$, while a minority $(\sim 15 \%)$ is distributed over the remaining 12 subtypes, with relative prevalences between $<1$ and $5 \%$. These percentages may differ considerably within certain ethnic groups, due to founder effects. All subtypes seem to fit within a "classical" FA phenotype, except for D1 and N (mutated in BRCA2/FANCD1 and PALB2/FANCN), which are associated with a distinct, more severe, syndromic association. The mode of inheritance for all subtypes is autosomal recessive, except for FA-B, which is X-linked. These two different modes of inheritance have important consequences for the counseling of FA families. The relative prevalence of FA-B amongst unselected FA patients is estimated at 1.6\% [5]. For all genetic subtypes disease genes have been identified (Table 1). Many mutations found in the various subtypes are private, but recurrent mutations are known, particularly in specific ethnic backgrounds (Table 2).

Most FA genes encode orphan proteins with no known molecular function. At least eight FA proteins (FANCA, $-\mathrm{B},-\mathrm{C},-\mathrm{E},-\mathrm{F},-\mathrm{G},-\mathrm{L}$, and $-\mathrm{M})$ assemble into a nuclear multiprotein core complex, which is required to activate FANCD2 and FANCI by monoubiquitination [6]. FANCL, which carries a RING finger domain, is supposed to represent the ubiquitin E3 ligase in this activation [7]. FANCM probably acts as a sensor of DNA damage and recruits the FA core complex to the site of damage, but FANCM also interacts with other proteins including Blm [6]. Monoubiquitination of FANCD2 and FANCI directs these proteins to areas of damaged chromatin where they interact with other proteins, resulting in repair of the damage [6]. The remaining FA 
TABLE 1: Fanconi anemia complementation groups, genes, and proteins.

\begin{tabular}{lllll}
\hline Group & Gene symbol $(\mathrm{s})^{\mathrm{a}}$ & Cytogenetic location & Protein (amino acids) & Domain structure (references) \\
\hline $\mathrm{A}$ & FANCA & $16 \mathrm{q} 24.3$ & 1455 & HEAT repeats [8] \\
$\mathrm{B}$ & FANCB & $\mathrm{Xp} 22.31$ & 859 & - \\
$\mathrm{C}$ & FANCC & $9 \mathrm{q} 22.3$ & 558 & HEAT repeats [8] \\
D1 & BRCA2 & $13 \mathrm{q} 12.3$ & 3418 & RAD51- and DNA-binding motifs [9] \\
$\mathrm{D} 2$ & FANCD2 & $3 \mathrm{p} 25.3$ & 1451 & - \\
$\mathrm{E}$ & FANCE & $6 \mathrm{p} 21.3$ & 536 & - \\
$\mathrm{F}$ & FANCF & $11 \mathrm{p} 15$ & 374 & - \\
$\mathrm{G}$ & FANCG & $9 \mathrm{p} 13$ & 622 & Tetratricopeptide repeats (TPR) [10] \\
$\mathrm{I}$ & FANCI & $15 \mathrm{q} 26.1$ & 1328 & - \\
$\mathbf{J}^{\mathrm{b}}$ & BRIP1 & $17 \mathrm{q} 22$ & 1249 & DNA helicase [11, 12] \\
$\mathrm{L}$ & FANCL & $2 \mathrm{p} 16.1$ & 375 & RING finger motif (E3 ligase) [7, 8] \\
$\mathrm{M}^{\mathrm{b}}$ & FANCM & $14 \mathrm{q} 21.3$ & 2014 & DNA helicase, nuclease [13] \\
$\mathbf{N}^{\mathrm{b}}$ & PALB2 & $16 \mathrm{p} 12.1$ & 1186 & - \\
$\mathbf{O}^{\mathrm{b}}$ & RAD51C & $17 \mathrm{q} 25.1$ & 376 & - \\
$\mathbf{P}^{\mathrm{b}}$ & SLX4 & $16 \mathrm{p} 13.3$ & 1834 & Endonuclease scaffold [3, 4] \\
\hline
\end{tabular}

${ }^{\mathrm{a}}$ For gene nomenclature see http://www.genenames.org/.

${ }^{\mathrm{b}}$ The proteins defective in groups D1, J, N, O, and P (boldface) act downstream or independent of the monoubiquitination of FANCD2; all other FA proteins act upstream of this process.

TABLE 2: Major recurrent mutations in FA.

\begin{tabular}{|c|c|c|c|c|}
\hline Gene & Mutation* & $\begin{array}{l}\text { Geographic/ethnic } \\
\text { background }\end{array}$ & Comment & References \\
\hline \multirow{4}{*}{ FANCA } & c.3788_3790del (p.Phe1263del) & European, Brazilian & Relatively mild & {$[14,15]$} \\
\hline & c.1115_1118delTTGG (p.Val372fs) & European & Relatively mild & {$[16]$} \\
\hline & $\begin{array}{l}\text { Exon } 12-17 \mathrm{del} \\
\text { Exon } 12-31 \mathrm{del}\end{array}$ & South-African & $\begin{array}{l}\text { Relatively common in } \\
\text { Afrikaners }\end{array}$ & {$[17]$} \\
\hline & c.295C>T (p.Gln99X) & $\begin{array}{l}\text { Spanish Gypsy } \\
\text { population }\end{array}$ & $\begin{array}{l}\text { Worldwide highest } \\
\text { prevalence of mutant } \\
\text { FANCA allele }\end{array}$ & {$[18]$} \\
\hline \multirow[t]{2}{*}{ FANCC } & c. $711+4 \mathrm{~A}>\mathrm{T}$ (originally reported as IVS4+4A $>\mathrm{T}$ ) & $\begin{array}{l}\text { Homozygous in } 80 \% \\
\text { of Ashkenazi Jewish } \\
\text { FA; relatively } \\
\text { common in Japan. }\end{array}$ & $\begin{array}{l}\text { Severe phenotype in Jews, } \\
\text { milder in Japanese. }\end{array}$ & {$[19-22]$} \\
\hline & c.67delG (originally reported as $322 \mathrm{delG}$ ) & $\begin{array}{l}\text { Homozygous in } \\
\text { approx. } 50 \% \text { of Dutch } \\
\text { FA patients }\end{array}$ & $\begin{array}{l}\text { Like other exon } 1 \\
\text { mutations, relatively mild } \\
\text { phenotype. }\end{array}$ & $\begin{array}{l}{[19,23-} \\
25]\end{array}$ \\
\hline FANCD2 & c. $1948-16 \mathrm{~T}>\mathrm{G}$ & Turkish & Founder mutation & {$[26]$} \\
\hline \multirow{6}{*}{ FANCG } & c.313G > T (p.Glu105X) & European & $\begin{array}{l}44 \% \text { of mutated FANCG } \\
\text { alleles in Germany. }\end{array}$ & {$[27]$} \\
\hline & c. $1077-2 A>G$ & Portuguese/Brazilian & Founder mutation & {$[27,28]$} \\
\hline & c. $1480+1 \mathrm{G}>\mathrm{C}$ & French-Canadian & Founder mutation & {$[28]$} \\
\hline & c. $307+1 \mathrm{G}>\mathrm{C}$ & Japanese & Founder mutation & {$[28,29]$} \\
\hline & c.1794_1803del (p.Trp599fs) & European & & {$[28]$} \\
\hline & c.637_643del (p.Tyr213fs) & Sub-Saharan Africa & $82 \%$ of all black FA patients & {$[30]$} \\
\hline FANCJ & c.2392C > T (p.Arg798X) & & $\begin{array}{l}\text { Found in ca. } 50 \% \text { of FA-J } \\
\text { patients of diverse ancestry; } \\
\text { ancient mutation or hot } \\
\text { spot. }\end{array}$ & {$[11,12]$} \\
\hline
\end{tabular}

Nucleotide numbering based on ATG $=+1$.

Published sequence variations in FA genes, with their descriptions conforming to the current nomenclature rules, are listed at http://www.rockefeller.edu/fanconi/. 
proteins function downstream of or parallel to the FANCD2 activation step [6]. The exact nature of the DNA damage response, which when defective causes FA, remains to be defined. FANCJ/BRIP1 and FANCM possess DNA helicase motifs, which strongly suggests that the FA pathway acts through a direct interaction with DNA, presumably to resolve or remodel blocked DNA replication forks resulting from DNA interstrand cross-link damage [6]. This idea is strengthened by the recent extension of the FA pathway with SLX4, a scaffold protein for structure-specific endonucleases involved in unhooking the DNA cross-link $[3,4]$.

\section{Laboratory Diagnostics in FA}

Cells derived from FA patients are-by definitionhypersensitive to chromosomal breakage induced by DNA cross-linking agents such as mitomycin C (MMC) or diepoxybutane (DEB) [31]. This cellular phenotype is ascertained using stimulated blood $\mathrm{T}$ lymphocytes. The indications for FA laboratory testing are rather broad [32]. As a consequence, in only a small proportion of patients (about 10\%) the chromosomal breakage test is positive, and an FA diagnosis is established. Since mutation testing by Sanger sequencing and MLPA is rather laborious, time consuming and therefore expensive, a positive chromosomal breakage test is a prerequisite for starting mutation screening. Confirmation of the FA diagnosis at the DNA level is important in patients in whom the chromosomal breakage test was inconclusive. Furthermore, knowledge about the FA subtype is relevant for the treatment and prognosis of the patients. In addition, identification of mutations allows carrier testing in the family and will enable prenatal DNA testing and preimplantation genetic diagnosis (PGD) in future pregnancies. Finally, this information can be used to rule out FA in potential donors for bone marrow transplantation.

Although simultaneous testing of all FA genes by next generation sequencing will be possible in the near future, this technique will not be available immediately for all laboratories worldwide. In addition, in populations with strong founder mutations, a limited test using Sanger sequencing and MLPA will be a cost-effective alternative [33]. The strategy outlined below has been developed at our DNA diagnostics laboratory to provide a molecular diagnosis of FA. It is recognized that mutations in FANCA account for $60-70 \%$ of all FA cases and that about $15-20 \%$ of the mutations in this gene are large deletions $[33,34]$. Therefore, DNA testing usually starts with a screen for deletions in FANCA. However, depending on the circumstances strategies may differ from case to case.

2.1. Materials. Genomic DNA (from e.g., leukocytes or fibroblasts derived from the proband or the parents) is adequate for most mutation screening assays. Screening on cDNA is more efficient but has several drawbacks: for high-quality cDNA, growing cells (stimulated leukocytes, lymphoblastoid cell lines, or fibroblasts) are necessary. In addition, common alternative splice variants will hamper the evaluation of DNA sequences. Therefore, screening on gDNA is the preferred method for mutation screening. However, during the diagnostic process, growing cells from the proband will be helpful in a couple of situations. Growing cells are indispensable for studying the effect of unclassified variants on splicing or to verify the disease gene by functional complementation of the cellular phenotype with a construct expressing a wild type copy of the suspected gene [35-37]. Finally, if no mutations can be de detected, growing cells can be used to reconfirm the diagnosis FA by checking MMC sensitivity in cell growth or G2-arrest assays $[38,39]$.

\subsection{Mutation Screening Strategy}

2.2.1. Hints from Ethnic Background or Phenotype. Information on the ethnic background of the proband may provide a clue for a specific pathogenic mutation that most likely causes the disease, such as c.711+4A>T (IVS4 + 4A $>\mathrm{T}$ ) in FANCC, a mutation present in homozygous state in $80 \%$ of all FA cases of Ashkenazi Jewish ancestry, and c.295C $>$ T in FANCA, which was present homozygously in all $40 \mathrm{FA}$ cases of Spanish Gypsy ancestry so far investigated. More examples of recurrent mutations are shown in Table 3. The distinct clinical phenotype of D1 and N patients (severely affected, often combined with leukemia or solid tumors below the age of 5 years) may provide a clue to favor BRCA2/FANCD1 and PALB2/FANCN as the first gene to be screened [4044]. This is especially worthwhile if confirmed by the cellular phenotype: in contrast to cells from all other known FA subtypes, cells from $\mathrm{D} 1, \mathrm{~N}$ and $\mathrm{O}$ patients are unable to form RAD51 foci upon exposure to X rays or MMC [43-45].

\subsubsection{No Clues Available}

(1) In the absence of any clue to the disease gene, mutation screening starts with a search for deletions in FANCA, as this type of mutation accounts for $40 \%$ of all mutant FANCA alleles. The quantitative multiplex ligation-dependent probe amplification (MLPA) method [46] is used for this initial screen, which identifies FANCA as the most likely disease gene in 1 out of 4 patients by the detection of ausually hemizygous-deletion. In parallel, the FANCA gene is completely sequenced. The combination of these two approaches identifies $60-70 \%$ of all FA patients as FA-A.

(2) Next, FANCC, $-E$, $-F$, and $-G$ are screened by DNA sequencing.

(3) Only if the proband is a male, FANCB is screened by MLPA and DNA sequencing,

In Table 4, optimized conditions are provided for the PCR amplification of FANCA, $-C,-E,-F,-G$, and $-B$. Most PCRs can be performed under standard conditions. The PCR primers have M13 extensions which allow sequencing of all fragments with universal sequencing primers. MLPA was performed according to the instructions of the supplier. Detailed information about the sequences of the MLPA probes is available from the website of the 
TABLE 3: Mutations detected in a cohort of 54 patients by screening FANCA, FANCC, FANCE, FANCF and FANCG.

\begin{tabular}{|c|c|c|c|c|c|c|c|c|}
\hline & \multirow[b]{2}{*}{$\begin{array}{l}\text { Country } \\
\text { of origin }^{1}\end{array}$} & \multirow[b]{2}{*}{ Gene } & \multicolumn{2}{|c|}{ Allele 1} & \multicolumn{4}{|c|}{ Allele 2} \\
\hline & & & DNA change & Effect & $\begin{array}{l}\text { Number of } \\
\text { database entries }\end{array}$ & DNA change & Effect & $\begin{array}{l}\text { Number of } \\
\text { database entries }\end{array}$ \\
\hline 1 & ES & FANCA & ex16_17del & del & $12 x$ & c.1115_1118del & p.Val372fs & $62 x$ \\
\hline 2 & PT & FANCA & c. $718 \mathrm{C}>\mathrm{T}$ & p.Gln240X & $2 \mathrm{x}$ & c. $2870 \mathrm{G}>\mathrm{A}$ & W957X & $1 \mathrm{x}$ \\
\hline 3 & NL & FANCA & ex15del & del & $3 x$ & ex15del & del & $3 x$ \\
\hline 4 & NL & FANCA & c.3788_3790del & p.Phe1263del & $215 x$ & c.3788_3790del & p.Phe1263del & $215 x$ \\
\hline 5 & $\mathrm{CA}$ & FANCA & c. $718 \mathrm{C}>\mathrm{T}$ & pGlnx240X & $2 \mathrm{x}$ & c. $1085 \mathrm{~T}>\mathrm{C}$ & p.Leu362Pro & novel \\
\hline 6 & PT & FANCA & c.3788_3790del & p.Phe1263del & $215 x$ & c. $4130 \mathrm{C}>\mathrm{G}$ & p.Ser1377X & $1 \mathrm{x}$ \\
\hline 7 & IE & FANCA & c.2812_2830dup & p.Asp944fs & $3 x$ & c.2812_2830dup & p.Asp944fs & $3 \mathrm{x}$ \\
\hline 8 & $\mathrm{AU}$ & FANCA & c. $2303 \mathrm{~T}>\mathrm{C}$ & p.Leu768Pro & $5 x$ & c. $2303 \mathrm{~T}>\mathrm{C}$ & p.Leu768Pro & $5 \mathrm{x}$ \\
\hline 9 & NL & FANCA & c. $862 \mathrm{G}>\mathrm{T}$ & p.Glu288X & $1 \mathrm{x}$ & c. $862 \mathrm{G}>\mathrm{T}$ & p.Glu288X & $1 \mathrm{x}$ \\
\hline 10 & NL & FANCA & ex11_33del & del & $1 x$ & c.2121delC & p.Asn707fs & novel \\
\hline 11 & DK & FANCA & ex1_8del & del & $1 \mathrm{x}$ & c.3788_3790del & p.Phe1263del & $215 x$ \\
\hline 12 & UK & FANCA & c.337_338del & p.Ala114fs & $1 \mathrm{x}$ & c. $3349 \mathrm{~A}>\mathrm{G}$ & p.Arg1117Gly & $2 \mathrm{x}$ \\
\hline 13 & UK & FANCA & c. $3568 \mathrm{C}>\mathrm{T}$ & p.Gln1190X & novel & c. $3568 \mathrm{C}>\mathrm{T}$ & p.Gln1190X & novel \\
\hline 14 & NL & FANCA & c. $487 \mathrm{delC}$ & p.Arg163fs & $1 \mathrm{x}$ & c. $2851 \mathrm{C}>\mathrm{T}$ & p.Arg951Trp & $11 x$ \\
\hline 15 & SE & FANCA & c. $88 \mathrm{delG}$ & p.Val30fs & novel & c. $100 \mathrm{~A}>\mathrm{T}$ & p.Lys34X & $2 \mathrm{x}$ \\
\hline 16 & NL & FANCA & c. $862 \mathrm{G}>\mathrm{T}$ & p.Glu288X & $9 x$ & c. $1771 \mathrm{C}>\mathrm{T}$ & p.Arg591X & $9 \mathrm{x}$ \\
\hline 17 & PT & FANCA & c.1709_1715+4del & p.Glu570fs & novel & c. $3430 \mathrm{C}>\mathrm{T}$ & p.Arg1144Trp & novel \\
\hline 18 & $\mathrm{NO}$ & FANCA & c. $100 \mathrm{~A}>\mathrm{T}$ & p.Lys34X & $2 \mathrm{x}$ & c. $1378 \mathrm{C}>\mathrm{T}$ & p.Arg460X & novel \\
\hline 19 & PT & FANCA & ex15_17del & del & $2 \mathrm{x}$ & ex15_17del & del & $2 \mathrm{x}$ \\
\hline 20 & NL & FANCA & c. $2982-192 A>G$ & splice $^{2}$ & novel & ex7_31del & del & \\
\hline 21 & $\mathrm{AU}$ & FANCA & c.427-8_427-5del & splice & novel & c. $1771 \mathrm{C}>\mathrm{T}$ & p.Arg591X & $9 \mathrm{x}$ \\
\hline 22 & $\mathrm{AU}$ & FANCA & c. $3491 \mathrm{C}>\mathrm{T}$ & p.Pro1164Leu & novel & c. $3491 \mathrm{C}>\mathrm{T}$ & p.Pro1164Leu & novel \\
\hline 23 & $\mathrm{CA}$ & FANCA & ex4_29del & del & novel & ex31del & del & $6 \mathrm{x}$ \\
\hline 24 & $\mathrm{NL}$ & FANCA & c. $3391 \mathrm{~A}>\mathrm{G}$ & p.Thr1131Ala & $15 x$ & c. $3391 \mathrm{~A}>\mathrm{G}$ & p.Thr1131Ala & $15 \mathrm{x}$ \\
\hline 25 & GR & FANCA & c. $2 \mathrm{~T}>\mathrm{C}$ & p.Met1? & $1 \mathrm{x}$ & c.3788_3790del & p.Phe1263del & $215 x$ \\
\hline 26 & IE & FANCA & c.851dup & p.Val285fs & novel & c. $2534 \mathrm{~T}>\mathrm{C}$ & p.Leu845Pro & $4 \mathrm{x}$ \\
\hline 27 & NL & FANCA & c. $2852 \mathrm{G}>\mathrm{A}$ & p.Arg951Gln & $6 x$ & c. $3624 \mathrm{C}>\mathrm{T}$ & $\mathrm{p} .=($ splice $)$ & $2 \mathrm{x}$ \\
\hline 28 & $\mathrm{AU}$ & FANCA & c.331_334dup & p.Leu112fs & novel & ex22_29del & del & novel \\
\hline 29 & NL & FANCA & c. $862 \mathrm{G}>\mathrm{T}$ & p.Glu288X & $9 x$ & c.3920delA & p.Gln1307fs & $2 \mathrm{x}$ \\
\hline 30 & IR & FANCA & ex21del & del & novel & ex21del & del & novel \\
\hline 31 & SE & FANCA & ex1_12del & del & novel & ex22_29del & del & novel \\
\hline 32 & NL & FANCB & c.755_767del & p.Leu252fs & novel & - & - & - \\
\hline 33 & NL & FANCC & c.67delG & p.Asp23fs & $50 \mathrm{x}$ & c. $553 \mathrm{C}>\mathrm{T}$ & p.Arg185X & $14 \mathrm{x}$ \\
\hline 34 & NL & FANCC & c.67delG & p.Asp23fs & $50 \mathrm{x}$ & c.67delG & p.Asp23fs & $50 x$ \\
\hline 35 & $\mathrm{CA}$ & FANCC & c.67delG & p.Asp23fs & $50 \mathrm{x}$ & c. $553 \mathrm{C}>\mathrm{T}$ & p.Arg185X & $14 \mathrm{x}$ \\
\hline 36 & NL & FANCC & c.67delG & p.Asp23fs & $50 x$ & c. $1155-1 \mathrm{G}>\mathrm{C}$ & splice & novel \\
\hline 37 & NL & FANCC & c.67delG & p.Asp23fs & $50 x$ & c.67delG & p.Asp23fs & $50 x$ \\
\hline 38 & NL & FANCC & c.67delG & p.Asp23fs & $50 \mathrm{x}$ & c. $467 \mathrm{delC}$ & p.Ser156fs & novel \\
\hline 39 & PT & FANCE & c. $1111 \mathrm{C}>\mathrm{T}$ & p.Arg371Trp & $6 x$ & c. $1111 \mathrm{C}>\mathrm{T}$ & p.Arg371Trp & $6 \mathrm{x}$ \\
\hline 40 & UK & FANCF & c. $496 \mathrm{C}>\mathrm{T}$ & p.Gln166X & $4 \mathrm{x}$ & c. $496 \mathrm{C}>\mathrm{T}$ & p.Gln166X & $4 \mathrm{x}$ \\
\hline 41 & UK & FANCG & c. $307+2$ delT & splice & novel & c. $307+2$ delT & splice & novel \\
\hline 42 & UK & FANCG & c.1471_1473delinsG & p.Lys491fs & novel & c.1471_1473delinsG & p.Lys491fs & novel \\
\hline 43 & NL & FANCG & c. $65 \mathrm{G}>\mathrm{C}$ & p.Arg22Pro & $6 x$ & c. $65 \mathrm{G}>\mathrm{C}$; & p.Arg22Pro & $6 \mathrm{x}$ \\
\hline 44 & IR & FANCG & c. $307+1 \mathrm{G}>\mathrm{C}$ & splice & $21 x$ & c. $307+1 \mathrm{G}>\mathrm{C}$ & splice & $21 x$ \\
\hline 45 & NL & FANCG & c. $85-1 \mathrm{G}>\mathrm{A}$ & splice & novel & c. $85-1 \mathrm{G}>\mathrm{A}$ & splice & novel \\
\hline
\end{tabular}

${ }^{1}$ Country of origins: AU: Australia; CA: Canada; DK: Denmark; ES: Spain; GR: Greece; IE: Ireland; IR: Iran; NL: Netherlands; PT: Portugal; SE: Sweden; UK: United Kingdom

Number of database entries refers to the FA database at: http://www.rockefeller.edu/fanconi/.

The pathogenic state of novel missense mutations is based upon in silico prediction algorithms (SIFT, POLYPHEN2, Align GVGD), the presence of a second clearly pathogenic mutation in the same gene and segregation in the family.

${ }^{2}$ Effect c.2982-192A>G: by studying cDNA it was shown that the mutation created a new splice donor site resulting in an aberrant mRNA. 
TABLE 4: Primers and conditions for PCR on genomic DNA of the coding sequence plus intron/exon boundaries of FANCA, FANCC, FANCE, FANCF, FANCG, and FANCB.

(a)

\begin{tabular}{|c|c|c|}
\hline \multicolumn{3}{|l|}{$\overline{F A N C A}$} \\
\hline Primer name & Sequence $\left(5^{\prime}>3^{\prime}\right)$ & Product length (bp) \\
\hline FANCA_ex1F & gtaaaacgacggccag GCGCCTCCCCCAGGACCAACA & 362 \\
\hline FANCA_ex1R & caggaaacagctatga AGGCTCTGGCGGGAAGGGATCGG & \\
\hline FANCA_ex2F & gtaaaacgacggccag CTCTTCGGGAGGGTGTCGCTGGT & 328 \\
\hline FANCA_ex2R & caggaaacagctatga CTCTTCGGGAGGGTGTCGCTGGT & \\
\hline FANCA_ex3F & gtaaaacgacggccag GCCTGGCCTGGAGCTTGAAT & 392 \\
\hline FANCA_ex3R & caggaaacagctatga CGCAGGTTGAATCAGACGCTGTT & \\
\hline FANCA_ex4F & gtaaaacgacggccag TAAGGCATTTTAAACAGCAAGTC & 430 \\
\hline FANCA_ex4R & caggaaacagctatga TGCCAATAAATACTGAGCAAACT & \\
\hline FANCA_ex5F & gtaaacgacggccag AGTATTGTTTCAGGTAATTTGTT & 356 \\
\hline FANCA_ex5R & caggaaacagctatga TGAAGGTACTTCTTTCCAATCCA & \\
\hline FANCA_ex6F & gtaaaacgacggccag AGATGTGTTTCAGGCTCTAAGTT & 402 \\
\hline FANCA_ex6R & caggaaacagctatga GCAATGCAATCTAGTCTAGTACA & \\
\hline FANCA_ex7F & gtaaaacgacggccag TGGGATTTAGTTGAGCCTTACGTCTGC & 421 \\
\hline FANCA_ex7R & caggaaacagctatgaAAGGTGAATGGAAACACTTAAACTCATGTCA & \\
\hline FANCA_ex8F & gtaaaacgacggccag GTGGTCAGGTGAGCAGTAACTTC & 401 \\
\hline FANCA_ex8R & caggaaacagctatga TAAATAGGTACAAACAGCACGTT & \\
\hline FANCA_ex9F & gtaaaacgacggccag TTCTCTTGTGTGATGCAGGTATC & 332 \\
\hline FANCA_ex9R & caggaaacagctatga TGACCCACAGATTCATGAGGTAT & \\
\hline FANCA_ex10F & gtaaaacgacggccag TTTTGATTAAGGCCTACAGATTG & 406 \\
\hline FANCA_ex10R & caggaaacagctatga CCTCCTCCTCACGCACGTTATCG & \\
\hline FANCA_ex11F & gtaaaacgacggccag TTTCAAGTCTGTGGTTATAGTGG & 410 \\
\hline FANCA_ex11R & caggaaacagctatga AGACGTAAAAGAGGTCCTAGAAT & \\
\hline FANCA_ex12F & gtaaaacgacggccag CTGTGGGGCTGGTCCTTAACAAA & 236 \\
\hline FANCA_ex12R & caggaaacagctatga AGGCAGCATGACAAGTACTAGGC & \\
\hline FANCA_ex13F & gtaaaacgacggccag ACATTGGTTTGCTTGGATATTGA & 377 \\
\hline FANCA_ex13R & caggaaacagctatga CTGACAAAGAATGTTCCATCGAC & \\
\hline FANCA_ex14F & gtaaaacgacggccag TGCTGTAATTGCTGTGTAGTCTT & 411 \\
\hline FANCA_ex14R & caggaaacagctatga ACTCACATGACAGAGAATCAGGT & \\
\hline FANCA_ex15F & gtaaaacgacggccag ACTACAGCAGCCGCCCGGACACT & 430 \\
\hline FANCA_ex15R & caggaaacagctatga GCAGATCTGCAGGAGGCTCTTGG & \\
\hline FANCA_ex16F & gtaaaacgacggccag TCCCAGGCAGTTCCCAGACTAAC & 312 \\
\hline FANCA_ex16R & caggaaacagctatga AGCTGATGACAAATCCTCGTAGA & \\
\hline FANCA_ex17F & gtaaaacgacggccag ACCGCTCССТССТCACAGACTAC & 334 \\
\hline FANCA_ex17R & caggaaacagctatga AAGGCTGAAAAACTCAACTCAAG & \\
\hline FANCA_ex18F & gtaaaacgacggccag GCGCACAGCATGTGGGCCTTTAC & 397 \\
\hline FANCA_ex18R & caggaaacagctatga GCAGCTGCTAGAGGCCTTTTCGG & \\
\hline FANCA_ex19F & gtaaaacgacggccag GTGCACAAGAAGACTTCATAATG & 284 \\
\hline FANCA_ex19R & caggaaacagctatga AGTCCTTGCTTTCTACACAACTG & \\
\hline FANCA_ex20F & gtaaaacgacggccag CTTCTCTGTGTTGCAGCATATTC & 298 \\
\hline FANCA_ex20R & caggaaacagctatga AGAAGAAACCTGGAAGTAGTCAT & \\
\hline FANCA_ex21F & gtaaaacgacggccag ATAATAGATTTGGGGATTGTAAT & 255 \\
\hline FANCA_ex21R & caggaaacagctatga CAACAGACACTCAAGGTTAGGAA & \\
\hline FANCA_ex22F & gtaaaacgacggccag TGCAGTGAAGAGTCCTGTTGAGT & 305 \\
\hline FANCA_ex22R & caggaaacagctatga ACACACCAGCCTGATGTCACTAT & \\
\hline FANCA_ex23F & gtaaaacgacggccag CAGTCAGCAGGATCCGTGGAATC & 416 \\
\hline
\end{tabular}


(a) Continued.

\begin{tabular}{|c|c|c|}
\hline \multicolumn{3}{|l|}{ FANCA } \\
\hline Primer name & Sequence $\left(5^{\prime}>3^{\prime}\right)$ & Product length $(\mathrm{bp})$ \\
\hline FANCA_ex23R & caggaaacagctatga GGCCCTGGAACATCTGATACGAC & \\
\hline FANCA_ex24F & gtaaaacgacggccag CCTTCCTGCTGCTCCCGTCC & 229 \\
\hline FANCA_ex24R & caggaaacagctatga CAGACTTGGCCCAGCAAGAG & \\
\hline FANCA_ex25F & gtaaaacgacggccag CCGCTGGTGGTTGGATTAGCTGT & 296 \\
\hline FANCA_ex25R & caggaaacagctatga TTTCCAGGGCACTGAAGACGAAT & \\
\hline FANCA_ex26F & gtaaaacgacggccag AGCTTGGAAGAGGGCAGTCTGCT & 347 \\
\hline FANCA_ex26R & caggaaacagctatga CTCTTCTAATTTTATCAAACGAG & \\
\hline FANCA_ex27F & gtaaaacgacggccag AGACTGTCTCACAACAAACGAAC & 356 \\
\hline FANCA_ex27R & caggaaacagctatga CGGTCCGAAAGCTGCGTAAAC & \\
\hline FANCA_ex28F & gtaaaacgacggccag GTTGATGGTCTGTTTCCACCTGA & 401 \\
\hline FANCA_ex28R & caggaaacagctatga GAAGGAACGGTCACCTACGTGCT & \\
\hline FANCA_ex29F & gtaaaacgacggccag GACATGGAGGACTGCGTATGAGA & 411 \\
\hline FANCA_ex29R & caggaaacagctatga GTGGCTGTGATGACTGGAACGTG & \\
\hline FANCA_ex30F & gtaaaacgacggccag CCCGAGCCGCCAGTCTCAACCCA & 411 \\
\hline FANCA_ex30R & caggaaacagctatga AAAGGCAGACCCACCCTAAGCTA & \\
\hline FANCA_ex31F & gtaaaacgacggccag GATAAGCCTCCTTGGTCATGGTA & 406 \\
\hline FANCA_ex31R & caggaaacagctatga TGGCAATAAATATCTTAATAGCA & \\
\hline FANCA_ex32F & gtaaaacgacggccag TTCCTGTGCCAGCATACTGCTCT & 359 \\
\hline FANCA_ex32R & caggaaacagctatga GGGTGGGGACACACAGACAAGTA & \\
\hline FANCA_ex33F & gtaaaacgacggccag TGGGTTTCAGGGTGGTGGTTGCT & 356 \\
\hline FANCA_ex33R & caggaaacagctatga GAACCCTTTCCTCAGTAATTCAC & \\
\hline FANCA_ex34F & gtaaaacgacggccag CGCCCAGGGAAGCCGTTAAGTTT & 333 \\
\hline FANCA_ex34R & caggaaacagctatga GCGTTCTGAGAAGGCCACGAGAG & \\
\hline FANCA_ex35F & gtaaaacgacggccag TTCCTTCACTCTACTAGTTGTGG & 311 \\
\hline FANCA_ex35R & caggaaacagctatga TGAGATGGTAACACCCGTGATGG & \\
\hline FANCA_ex36F & gtaaaacgacggccag CCATCTCAGCCACCCTCATCTGT & 350 \\
\hline FANCA_ex36R & caggaaacagctatga AGGCGCCCACCACCACGAGAACT & \\
\hline FANCA_ex37F & gtaaaacgacggccag GACTTGGTTTCTATGGCGTGGTT & 310 \\
\hline FANCA_ex37R & caggaaacagctatga CCCAGAGAAATAGCACTGATTGA & \\
\hline FANCA_ex38F & gtaaaacgacggccag GTTTTCTAAGATCCACTTAAAGG & 362 \\
\hline FANCA_ex38R & caggaaacagctatga CTCACTCACACTTCCGCAAACAC & \\
\hline FANCA_ex39F & gtaaaacgacggccag CTGTCCAGAGGCCCAGTATTACC & 387 \\
\hline FANCA_ex39R & caggaaacagctatga AGGAGGGCTCGTTCTTAACCATT & \\
\hline FANCA_ex40F & gtaaaacgacggccag GGTGTCCCCAGCACTGATAATAG & 353 \\
\hline FANCA_ex40R & caggaaacagctatga AGACATAGTGACAAATGGCTACA & \\
\hline FANCA_ex41F & gtaaaacgacggccag CCCTTGGCATCACCTGCTACCTT & 403 \\
\hline FANCA_ex41R & caggaaacagctatga AACAGGCAAACTCACAGGTTAGA & \\
\hline FANCA_ex42F & gtaaaacgacggccag ACCAGCCCTGTTTCTGTATGTCT & 248 \\
\hline FANCA_ex42R & caggaaacagctatga ACATGGCCCAGGCAGCTGTCAAT & \\
\hline FANCA_ex43F & gtaaaacgacggccag TGTGGGGGACATGAGAATTGACA & 378 \\
\hline FANCA_ex43R & caggaaacagctatga GTAATCCACTTTTTAGTGCAACA & \\
\hline FANCAIVS10F & gtaaaacgacggccag TTTACATGTGCATCAGTTAGCTT & 184 \\
\hline FANCAIVS10R & caggaaacagctatga CATGAAGACACAGAAAAAGTAGGT & \\
\hline
\end{tabular}

(b)

\section{FANCC}

Primer name

FANCC_ex1F

Sequence $\left(5^{\prime}>3^{\prime}\right)$

Product length (bp)

FANCC_ex1R

gtaaaacgacggccag ACCATTTCCTTCAGTGCTGGACA caggaaacagctatga CCATCGGCACTTCAGTCAATACC 
(b) Continued.

\begin{tabular}{|c|c|c|}
\hline \multicolumn{3}{|l|}{ FANCC } \\
\hline Primer name & Sequence $\left(5^{\prime}>3^{\prime}\right)$ & Product length (bp) \\
\hline $\begin{array}{l}\text { FANCC_ex2F } \\
\text { FANCC_ex } 2 \mathrm{R}\end{array}$ & $\begin{array}{l}\text { gtaaaacgacggccag CTAAACAAGAAGCATTCACGTTC } \\
\text { caggaaacagctatga GGAGAAAGGTTCATAATGTAAGC }\end{array}$ & 303 \\
\hline $\begin{array}{l}\text { FANCC_ex3F } \\
\text { FANCC_ex3R }\end{array}$ & $\begin{array}{l}\text { gtaaaacgacggccag TCAGCAGAAAGAGAATGTGCAAA } \\
\text { caggaaacagctatga AACATCATAGAACTGGATTCCAC }\end{array}$ & 405 \\
\hline $\begin{array}{l}\text { FANCC_ex4F } \\
\text { FANCC_ex4R }\end{array}$ & $\begin{array}{l}\text { gtaaaacgacggccag TGTACATAAAAGGCACTTGCATT } \\
\text { caggaaacagctatga TCCCATCTCACATTTCTTCCGTA }\end{array}$ & 380 \\
\hline $\begin{array}{l}\text { FANCC_ex5F } \\
\text { FANCC_ex5R }\end{array}$ & $\begin{array}{l}\text { gtaaaacgacggccag AGAACTGATGTAATCCTGTTTGC } \\
\text { caggaaacagctatga TTACTGCTCTGTGAGAGTTGAGA }\end{array}$ & 367 \\
\hline $\begin{array}{l}\text { FANCC_ex6F } \\
\text { FANCC_ex6R }\end{array}$ & $\begin{array}{l}\text { gtaaaacgacggccag GTCTTTGACCTTTTTAGCATGAA } \\
\text { caggaaacagctatga AACGTTTGGACACTGCTGTCGTA }\end{array}$ & 387 \\
\hline $\begin{array}{l}\text { FANCC_ex7F } \\
\text { FANCC_ex7R }\end{array}$ & $\begin{array}{l}\text { gtaaaacgacggccag ATTAGTGATTGCATTTTGAACTT } \\
\text { caggaaacagctatga CAAAAATAAAATGTAAATACACG }\end{array}$ & 422 \\
\hline $\begin{array}{l}\text { FANCC_ex8F } \\
\text { FANCC_ex8R }\end{array}$ & $\begin{array}{l}\text { gtaaaacgacggccag CTCCTTTGGCTGATAATAGCAAG } \\
\text { caggaaacagctatga CTGATTTTTGAGTTTTTACCTCT }\end{array}$ & 336 \\
\hline $\begin{array}{l}\text { FANCC_ex9F } \\
\text { FANCC_ex9R }\end{array}$ & $\begin{array}{l}\text { gtaaaacgacggccag ATACTGCTGAAGCTTATGGCACA } \\
\text { caggaaacagctatga TAACCTTTGTTGGGGCACTCATT }\end{array}$ & 400 \\
\hline $\begin{array}{l}\text { FANCC_ex10F } \\
\text { FANCC_ex10R }\end{array}$ & $\begin{array}{l}\text { gtaaaacgacggccag TATGAGGTTATTGGGAGCTTATT } \\
\text { caggaaacagctatga CTGTCTCCCTCATGCTGTAGATA }\end{array}$ & 382 \\
\hline $\begin{array}{l}\text { FANCC_ex } 11 \mathrm{~F} \\
\text { FANCC_ex11R }\end{array}$ & $\begin{array}{l}\text { gtaaaacgacggccag GAACCAGAAGTAAAGGGCGTCTC } \\
\text { caggaaacagctatga CTGACCTGCTCCAAGCCATCCGT }\end{array}$ & 416 \\
\hline $\begin{array}{l}\text { FANCC_ex12F } \\
\text { FANCC_ex12R }\end{array}$ & $\begin{array}{l}\text { gtaaaacgacggccag AAGTACAATTTAAGCCAACCGTT } \\
\text { caggaaacagctatga AGGTTGCCATGACATATGCCATC }\end{array}$ & 451 \\
\hline $\begin{array}{l}\text { FANCC_ex13F } \\
\text { FANCC_ex13R }\end{array}$ & $\begin{array}{l}\text { gtaaaacgacggccag CCTCTCTCAGGGGCCAGTGCTTA } \\
\text { caggaaacagctatga AGACCCTCGGACAGGTAACCCAC }\end{array}$ & 435 \\
\hline $\begin{array}{l}\text { FANCC_ex14F } \\
\text { FANCC_ex14R }\end{array}$ & $\begin{array}{l}\text { gtaaaacgacggccag ACTTGCTATGCTAATCACCTTGC } \\
\text { caggaaacagctatga AATGCGTGGCCACAGGTCATCAC }\end{array}$ & 437 \\
\hline
\end{tabular}

(c)

\begin{tabular}{llc}
\hline FANCE & & Product length $(\mathrm{bp})$ \\
Primer name & Sequence $\left(5^{\prime}>3^{\prime}\right)$ & 540 \\
\hline FANCE_ex1F & $\begin{array}{l}\text { gtaaaacgacggccag CGCCTCCCTCCTTCCCTTTC } \\
\text { caggaaacagctatga CCCGCCTCCCATACCTGCTAA } \\
\text { FANCE_ex1R } \\
\text { gtaaaacgacggccag GCTCTGCCCAGTCTGCCTTGTGC } \\
\text { FANCE_ex2aF }\end{array}$ & 469 \\
FANCE_ex2aR & gtaaaacgacggccag GCCAGAGACAGCTCCAAAGTCTA \\
FANCE_ex2bF & caggaaacagctatga CAGCCTTCCCCATGGATAAAGCC \\
FANCE_ex2bR & gtaaaacgacggccag GCCTCTTGACTTTCTTGAATCAT \\
FANCE_ex3F & caggaaacagctatga ACTGTCCTCAGACCTTTACTCCA & 479 \\
FANCE_ex3R & gtaaaacgacggccag TTGAACCAAGTGTAGACTTACCA \\
FANCE_ex4F & caggaaacagctatga GGGAAGGAACCAAGGGCTAAAAG \\
FANCE_ex4R & gtaaaacgacggccag GTATCTTTTAGCCCTTGGTTCCT \\
FANCE_ex5F & caggaaacagctatga GAATCCCCTCTCTCAAGTACCAC \\
FANCE_ex5R & gtaaaacgacggccag TTTCCTTTGTAACATGTATCATC \\
FANCE_ex6F & caggaaacagctatga AGCAGAAAGCAGGGAGGCGGTAA & 352 \\
FANCE_ex6R & gtaaaacgacggccag ACAGGCTGGGCATTCTGTTACCG \\
FANCE_ex7F & caggaaaacagctatga AGTGAGACACAAGGATCCCCTAA \\
FANCE_ex7R & gtaaaacgacggccag TTGGAGCAGCAGATAGATACTCA \\
FANCE_ex8F & caggaaacagctatga AGAGGTGGAGCTGAAGTGACCAT \\
FANCE_ex8R & gtaaaacgacggccag GTTACCTGCCCAGGGTCACCTAG \\
FANCE_ex9F & caggaaacagctatga CTGGCCAGCACTCAGGGTTTTAT & 436 \\
FANCE_ex9R & & 431 \\
\hline
\end{tabular}


(c) Continued.

\section{FANCE}

Primer name

Sequence $\left(5^{\prime}>3^{\prime}\right)$

Product length (bp)

FANCE_ex10F

FANCE_ex10R

gtaaaacgacggccag TGGCCTCCTCTCTCCTCAATAGA

caggaaacagctatga AACAGGGAGGCAGTTGCAATCTG

369

(d)

\section{FANCF}

Primer name

FANCF_ex1aF

FANCF_exlaR

FANCF_ex $1 \mathrm{bF}$

FANCF_ex1bR

FANCF_exlcF

FANCF_exlcR

FANCF_exldF

FANCF_ex1dR

Sequence $\left(5^{\prime}>3^{\prime}\right)$

Product length (bp)

gtaaaacgacggccag TTTCGCGGATGTTCCAATCAGTA

caggaaacagctatga CTGCACCAGGTGGTAACGAGCTG

449

gtaaaacgacggccag AGTGGAGGCAAGAGGGCGGCTTT

caggaaacagctatga GCTATCACCTTCAGGAAGTTGTT

456

gtaaaacgacggccag CCCAAATCTCCAGGAGGACTCTC caggaaacagctatga TTTCTGAAGGTCATAGTGCAAAC

444

gtaaaacgacggccag GCTTTTGACTTTAGTGACTAGCC caggaaacagctatga ATTTGGTGAGAACATTGTAATTT

456

(e)

FANCG

Primer name

Sequence $\left(5^{\prime}>3^{\prime}\right)$

Product length (bp)

FANCG_ex1F

FANCG_ex1R

gtaaaacgacggccag AGCCTGGGCGGGTGGATTGGGAC

caggaaacagctatga TCATTTCTGGCTCTTTGGTCAAG

389

FANCG_ex2F

FANCG_ex2R

gtaaaacgacggccag CAGGCCAAGGTAACACGGTTGCT

caggaaacagctatga CCAGTCTCCTCTGTGCCTTAAAC

460

FANCG_ex3F

FANCG_ex3R

gtaaaacgacggccag TATTGTAGCTGTTTTGGTTGGAG

caggaaacagctatga GGTGACAGATGTTGTTTATCCTC

362

gtaaaacgacggccag GGAGATGGAGGATGAGGTGCTAC

caggaaacagctatga CGACCACCAACCCAGCCGCCTGT

411

FANCG_ex4R

gtaaaacgacggccag AGATGGAGATAGGAGAAGACGAG caggaaacagctatga GCTTCATGAAGGCTGCTTAGTGC

FANCG 5 5R

FANCG_ex6F

FANCG_ex6R

gtaaaacgacggccag CAGTTCCATGGGCTTCTTAGACC

caggaaacagctatga TCAGGGCTGCAACCAAGTACAAC

454

gtaaaacgacggccag GCACTGGGGTCCTGTCACCGTAA caggaaacagctatga ATAATCTTTGGGAGCCATACTTC

gtaaaacgacggccag GCTTGTGATGGGGTGACTTGACT caggaaacagctatga AGTTCAGGTCTAGAAGCAAGGTA

gtaaaacgacggccag CCTCCTCAGGGCCCATGAACATC

caggaaacagctatga GCAGTGTCTTGAAAGGCATGAGC

FANCG_ex7F

FANCG_ex7R

FANCG_ex8F

FANCG_ex8R

FANCG_ex9F

FANCG_ex9R

FANCG_ex10F

FANCG_ex10R

FANCG_ex11F

FANCG_ex11R

FANCG_ex12F

FANCG_ex12R

FANCG_ex13F

FANCG_ex13R

FANCG_ex14F

FANCG_ex14R gtaaaacgacggccag CAGGACTCTGCATGGTACCAG caggaaacagctatga CCAATCAGAAAATCATCCCTC

gtaaaacgacggccag AGCTCCATGTTCACCTACTTACC caggaaacagctatga CAGTGCCGCATCTGACTTACATC

gtaaaacgacggccag AGGATTTGGGGTTTTGGTGACTG caggaaacagctatga AACTCTTGGGAGCCCTGCATACA gtaaaacgacggccag CCGCTTCCATATGTGAGTGTAGG caggaaacagctatgaC CACAATAGGTCCAAGGACTCTA

gtaaaacgacggccag CCAAACTAAGGGGTCACATGAAG caggaaacagctatga GATGGTGAAGCAGAAAGCCCTCC
418

438

400

460

397

445

340

405

(f)

\section{FANCB}

Primer name

Sequence $\left(5^{\prime}>3^{\prime}\right)$

Product length (bp)

FANCB_ex3AF gtaaaacgacggccag

FANCB_ex3AR 
(f) Continued.

\begin{tabular}{lll}
\hline FANCB & & \\
Primer name & Sequence $\left(5^{\prime}>3^{\prime}\right)$ & Product length $(\mathrm{bp})$ \\
\hline FANCB_ex3BF & gtaaacgacggccag & \\
FANCB_ex3BR & ATTAACCTCCCTTACATTGTGATAGcaggaaacagctatga & 811 \\
& CAATAAGACTCCAGAATGAACTCTA & \\
FANCB_ex4F & gtaaaacgacggccag & \\
FANCB_ex4R & TTTACAAATGACAACTACATGAcaggaaacagctatga \\
FANCB_ex5F & gtaaaacgacggccag ACTGCATCTGGCCTATAGTTcaggaaacagctatga \\
FANCB_ex5R & AATACCATTTTTACCCAAGC \\
FANCB_ex6F & gtaaaacgacggccag GTATTTCCTGAATTATTGGTATGTC \\
FANCB_ex6R & caggaaacagctatga CATAAAAGTCCACCATTATAACCTC \\
FANCB_ex7F & gtaaaacgacggccag TGTTTGGGCCATAAGCCCTA \\
FANCB_ex7R & caggaaacagctatga TTCTGGAGCATCAAGACAGT \\
FANCB_ex8F & gtaaaacgacggccag GTTGTTTGTATGACATTTAATCATC \\
FANCB_ex8R & caggaaacagctatga ATCATTAAACTCTGCCCATTATCAG \\
FANCB_ex9F & gtaaaacgacggccag AGGTAATTTTGTTGGCACTT \\
FANCB_ex9R & caggaaacagctatga ATGCGTTCATTCATGCTAGG \\
FANCB_ex10F & gtaaaacgacggccag AATTGGTTCTGTTTATCATTATGGT \\
FANCB_ex10R & caggaaacagctatga CTACTACAGTAAGCCTCGGTGTTTA & 391 \\
\hline
\end{tabular}

PCR conditions:

PCR was performed in Applied Biosystems PE9700 system using 96-well plates. PCR reactions (final volume $25 \mu \mathrm{l}$ ) contained 0.5 units Platinum Taq polymerase (Invitrogen), $1,5 \mathrm{mM} \mathrm{MgCl}_{2}, 0.2 \mathrm{mM}$ NTPs (Invitrogen), and 10 pmol primer.

For the large majority of amplicons, standard PCR conditions were used: preheat $95^{\circ} \mathrm{C}, 5 \mathrm{~min}$, denaturation $95^{\circ} \mathrm{C}, 30 \mathrm{sec}$, annealing $60^{\circ} \mathrm{C}, 30 \mathrm{sec}$., elongation $72^{\circ} \mathrm{C}, 1 \mathrm{~min}$, number of cycles: 33 .

Fragments with a different annealing temperature were FANCA exons 5, 7, 13, 21, 26, 31, and 38, FANCC exon 7, FANCF fragment 1d and FANCE exon $1: 55^{\circ} \mathrm{C}$; FANCA exon $1: 64^{\circ} \mathrm{C}$. For FANCE exon 1 the PCR mix was supplemented with $10 \%$ DMSO.

For $F A N C B$ different PCR conditions were used: preheat $95^{\circ} \mathrm{C}, 5 \mathrm{~min}$, denaturation $95^{\circ} \mathrm{C}, 1 \mathrm{~min}$, annealing $50^{\circ} \mathrm{C}, 1 \mathrm{~min}$., elongation $72^{\circ} \mathrm{C}, 1 \mathrm{~min}$., number of cycles 30. For $F A N C B$ exon 7 and 9 the annealing temperature was $55^{\circ} \mathrm{C}$. For sequencing of exon 7 forward, a special sequencing primer was used: $5^{\prime}-$ TTTTTAGAAGGAATGTCTTG-3'.

FA gene specific part of the primer is indicated in capitals. Primers are extended with M13 sequence (indicated in normal letter type), which is used for the sequencing reaction.

supplier (www.mlpa.com). In a well-equipped laboratory with sufficient dedicated personal, testing of FANCA, $-C,-E$, $-F,-G$ and $-B$ can be completed within 1-2 weeks.

After screening FANCA, $-C,-E,-F,-G$, and $-B$, a molecular diagnosis is obtained for $\sim 85 \%$ of the patients [34]. In our cohort of 54 patients, referred to our diagnostic service since 2008 , mutations were detected in 45 patients (83\%). FANCA mutations were found in 31 of the patients (57\%), FANCC mutations in 6 patients $(11 \%)$, and FANCG mutations in 5 patients (9\%). FANCB, FANCE, and FANCF mutations were found in single families (Table 3 ). In the small group of patients without mutations no complementation analysis or FANCD2 western blotting was performed. Therefore, we do not know if we missed FANCA, $-C,-E,-F,-G$, and $-B$ mutations in these patients or that these patients have mutations in other FA genes. Table 3 does not include prenatal cases, because prenatal testing is only offered in couples in which the FA-causing mutations are already established. Testing was offered as a diagnostic service for which a fee was charged.

For the patients negative for FANCA, $-C,-E,-F,-G$, and $-B$ mutations, next generation sequencing can be used to analyze all other FA genes. If this technique is not available, further analysis will depend on the availability of growing cells from the proband. In that case a western blot should reveal whether both FANCD2 isoforms are present at normal levels.

(1) If both FANCD2 bands are absent or very weak, $F A N C D 2$ is sequenced. Because of the presence of FANCD2 pseudogene sequences in the genome, this testing must be performed on cDNA or gDNA using specially designed primers [26].

(2) If only the short isoform of FANCD2 is present, FANCL and FANCM are sequenced. If no mutations are found, the patient may be mutated in FANCI or in another unidentified FA gene acting upstream of FANCD2.

(3) If both isoforms are present, and if the clinical phenotype is compatible with FA-D1 or FA-N, BRCA2/FANCD1 and PALB2/FANCN are screened by MLPA and DNA sequencing.

(4) If negative, BRIP1/FANCJ, PALB2/FANCN, RAD51C/ FANCO, and SLX4/FANCP are sequenced.

(5) If negative again, the patient should be screened for mutations in NBS1, ESCO2 and DDX11 to test for Nijmegen breakage syndrome, Roberts syndrome and Warsaw Breakage syndrome, respectively $[47,48]$. The latter two syndromes can also be 
excluded by analyzing metaphase spreads for sister chromatid cohesion defects. If again negative, the patient is likely to be mutated in a novel FA gene acting downstream of FANCD2 ubiquitination.

\section{Notes}

3.1. Mutation Screening in Mosaic Patients. If an available lymphoblastoid cell line from an FA patient is phenotypically normal due to genetic reversion at the disease locus, mutation screening is still possible in the reverted cell line, since at least one mutation will be present [49-51]. The second mutation may be identified through investigating the parents.

3.2. Unclassified Variants. Missense mutations or in-frame deletions or insertions should be judged using in silico prediction algorithms (SIFT, POLYPHEN2, Align GVGD). Alternatively, they can be tested for pathogenicity in a cellular transfection assay to check the ability of the variant gene product to complement the cellular FA defect in a deficient cell line (see e.g., $[10,35,52])$. Generally, these tests are only feasible in a setting where a diagnostic laboratory is equipped with a research laboratory with all necessary technology.

\subsection{Functional Assignment to Genetic Subtypes. Retroviral} constructs have been used to identify the FA subtype by functional complementation, as an intermediate step before a mutation screen is undertaken [36]. Although knowing the disease gene facilitates mutation screening, retroviral transduction has some drawbacks in comparison to direct mutation screening: (i) growing, MMC-sensitive cells either from a cell line or fresh blood sample are required, which are not always easy to obtain; (ii) overexpression of some FA proteins (e.g., FANCM and FANCP) may be toxic for cells; (iii) novel genetic subtypes that emerge after all known groups have been excluded and cannot be readily distinguished from false negatives, that is, transductions that for some unknown reason have failed to cause complementation; (iv) the method requires relatively advanced laboratory facilities and technology. However, functional assignment of complementation group can rapidly be provided by laboratories with capability for this type of analysis [37], which has greatly facilitated reliable genotyping for over $95 \%$ of FA patients for which viral constructs are available.

3.4. Genetic Counseling. All patients with a diagnosis of FA confirmed by mutation analysis should be referred for genetic counselling, together with their parents and siblings. Mutation testing should be performed in all sibs regardless of any clinical symptoms. A complete pedigree, including a cancer history anamnesis, should be prepared. Mutation carriers might be at increased cancer risk (see Section 3.7) whose aspect should be included in the counseling (see Section 3.7).

FA patients themselves usually have decreased fertility. Women usually have late menarche, irregular menses, and early menopause. However, pregnancies in women with
FA have been described, and therefore women should be adequately informed about the risks for their offspring, which is mainly related to an increase in pregnancy-related complications [53].

Sibs of the parents of an FA patient often request carrier screening to assess their risk of getting a child with FA. If a sib appears to be carrier, this risk is still minimal because of the very low carrier frequency in the population. In the US the carrier frequency has been estimated to be about 1 in 181 [54]. The risk of a proven carrier to get a child with FA is therefore about 1 in 724 . However, in small communities or in consanguineous couples this risk is much higher, and mutation screening in spouses of proven carriers may be indicated.

3.5. Prenatal Diagnosis. Prenatal diagnosis of FA is relatively straightforward after the pathogenic mutations in a given family have been identified. Fetal cells can be obtained by chorionic villus sampling (CVS) during weeks 10-12 of the pregnancy or by amniocentesis, which is performed between weeks 14 and 16. However, CVS may be preferred as the diagnosis will be known at an earlier stage. If the mutation is not known, a chromosomal breakage test on fetal material may be performed [55], but this test may be considered less reliable than screening for mutations in the fetal material. Alternatively, flow cytometric testing of MMC sensitivity in amniotic cell cultures might be an option; however this technique is only available in a limited number of specialized laboratories [56]. Occasionally, FA may be suspected by fetal ultrasound imaging and confirmed by parental carrier testing when the family is not yet known to carry a risk for FA [57].

3.6. Genotype-Phenotype Correlation. FA is considered as one disease, and the question may be raised whether all fifteen genetic subtypes equally conform to the clinical FA phenotype. Genotype-phenotype correlation studies comparing the 3 most common groups A, C, and G indicated modest phenotypic differences, which were rather correlated with the relative severity of the mutations [23]. However, bias due to the ethnic distribution of the studied population is very well possible. Other studies reported significant differences between FA-A/G versus FA-C [58]. Cases in group FAD1 (mutated in BRCA2) and FA-N (mutated in PALB2) present with a distinct, relatively severe, phenotype that is characterized by the development of leukemia at very young age (median 2.2 years) and by pediatric cancers such as nephroblastoma (Wilms tumor) or medulloblastoma [4044]. The observations that one of the pathogenic mutations in BRCA2 in FA-D1 patients is hypomorphic and that mice with biallelic null alleles in Brca2 are embryonic lethals suggest that the BRCA2 protein serves a function that is essential for survival.

Different mutations in the same gene may be associated with divergent phenotypes, as illustrated by the two FANCC mutations, c.711+4A $>\mathrm{T}$ and c.67delG. The former (splicesite) mutation is associated with a relatively severe phenotype in Ashkenazi Jewish people [19] although the associated phenotype was reportedly less severe in patients of Japanese 
ancestry [20]. The carrier frequency for this mutation in the Ashkenazi population is relatively high (1 in 87), which has led to the recommendation of carrier detection to prevent disease [59]. In the Netherlands more than 50\% of FA cases are homozygous for the FANCC frameshift mutation c.67delG. The phenotype associated with this mutation, like other exon 1 mutations, seems relatively mild, as these patients rarely have skeletal abnormalities and show a relatively late age of onset of their marrow failure [24]. Awareness of such genetically determined phenotypic differences may help in clinical decision making, including the counselling of patients and families.

3.7. Cancer Risk in Heterozygous Mutation Carriers. An important issue is whether FA mutation carriers are at increased risk to develop cancer or other types of disease. Overall, there is no increased risk for cancer among FA heterozygotes $[60,61]$. However, the situation is different in some of the less prevalent FA subtypes. The FA-D1 subtype is caused by mutations in BRCA2 [62] which is a well-known breast and ovarian cancer predisposition gene [63]. In FA-D1 one of the mutations will be hypomorphic because biallelic "severe" mutations are supposed to be lethal [26]. Therefore, one of the parents of a FA-D1 patient will be a heterozygous carrier of a "severe" inactivating BRCA2 mutation and may thus have an increased risk for breast cancer and other BRCA2-associated cancers. Whether the parent with the hypomorphic mutation is also at increased risk is unknown: in breast cancer families these hypomorphic mutations are considered as variants with unknown clinical significance. Two other genes involved in FA and related to breast or ovarian cancer predisposition are PALB2/FANCN [64, 65] and RAD51C/FANCO [66]. Although cancer patients have been identified with germ-line mutations in these genes, an accurate estimate of the relative cancer risk for mutation carriers is still lacking.

Another special case is represented by female FANCB mutation carriers, who are supposed to consist of 50\% FA-like cells due to silenced expression of the wild type $F A N C B$ allele by the random process of $\mathrm{X}$ inactivation that occurs during early embryonic development. Nevertheless, in the few female FANCB mutation carriers studied so far, inactivation appeared strongly skewed towards the mutated allele [67]. This suggests that FA cells have a poor chance to survive next to unaffected cells in the same tissue, and these FA cells may therefore not give an increased cancer risk. However, the data are scarce at present so that no firm conclusions can be drawn regarding the cancer risk of female FANCB mutation carriers [60].

\section{Conflict of Interests}

The authors do not declare any conflict of interests related to this study.

\section{Acknowledgments}

The authors thank the Fanconi Anemia Research Fund, Inc., Eugene, OR, the Netherlands Organization for Health and
Development, and the Dutch Cancer Society, for financial support.

\section{References}

[1] J. P. de Winter and H. Joenje, "The genetic and molecular basis of Fanconi anemia," Mutation Research, vol. 668, no. 1-2, pp. 11-19, 2009.

[2] F. Vaz, H. Hanenberg, B. Schuster et al., "Mutation of the RAD51C gene in a Fanconi anemia-like disorder," Nature Genetics, vol. 42, no. 5, pp. 406-409, 2010.

[3] C. Stoepker, K. Hain, B. Schuster et al., "SLX4, a coordinator of structure-specific endonucleases, is mutated in a new Fanconi anemia subtype," Nature Genetics, vol. 43, no. 2, pp. 138-141, 2011.

[4] Y. Kim, F. P. Lach, R. Desetty, H. Hanenberg, A. D. Auerbach, and A. Smogorzewska, "Mutations of the SLX4 gene in Fanconi anemia," Nature Genetics, vol. 43, no. 2, pp. 142-146, 2011.

[5] M. Levitus, H. Joenje, and J. P. de Winter, "The Fanconi anemia pathway of genomic maintenance," Cellular Oncology, vol. 28, no. 1-2, pp. 3-29, 2006.

[6] A. J. Deans and S. C. West, "DNA interstrand crosslink repair and cancer," Nature Reviews Cancer, vol. 11, no. 7, pp. 467480, 2011.

[7] A. R. Meetei, J. P. de Winter, A. L. Medhurst et al., "A novel ubiquitin ligase is deficient in Fanconi anemia," Nature Genetics, vol. 35, no. 2, pp. 165-170, 2003.

[8] E. Blom, "Evolutionary clues to the molecular function of Fanconi anemia genes," in Thesis, VU University Medical Center, Amsterdam, The Netherlands, 2006.

[9] S. C. West, "Molecular views of recombination proteins and their control," Nature Reviews Molecular Cell Biology, vol. 4, no. 6, pp. 435-445, 2003.

[10] E. Blom, H. J. van de Vrugt, Y. de Vries, J. P. de Winter, F. Arwert, and H. Joenje, "Multiple TPR motifs characterize the Fanconi anemia FANCG protein,” DNA Repair, vol. 3, no. 1, pp. 77-84, 2004.

[11] M. Levitus, Q. Waisfisz, B. C. Godthelp et al., "The DNA helicase BRIP1 is defective in Fanconi anemia complementation group J," Nature Genetics, vol. 37, no. 9, pp. 934-935, 2005.

[12] O. Levran, C. Attwooll, R. T. Henry et al., "The BRCA1interacting helicase BRIP1 is deficient in Fanconi anemia," Nature Genetics, vol. 37, no. 9, pp. 931-933, 2005.

[13] A. R. Meetei, A. L. Medhurst, C. Ling et al., "A human ortholog of archaeal DNA repair protein Hef is defective in Fanconi anemia complementation group M," Nature Genetics, vol. 37, no. 9, pp. 958-963, 2005.

[14] O. Levran, T. Erlich, N. Magdalena et al., "Sequence variation in the Fanconi anemia gene FAA," Proceedings of the National Academy of Sciences of the United States of America, vol. 94, no. 24, pp. 13051-13056, 1997.

[15] N. Magdalena, D. V. Pilonetto, M. A. Bitencourt et al., "Frequency of Fanconi anemia in Brazil and efficacy of screening for the FANCA 3788-3790del mutation," Brazilian Journal of Medical and Biological Research, vol. 38, no. 5, pp. 669-673, 2005.

[16] O. Levran, R. Diotti, K. Pujara, S. D. Batish, H. Hanenberg, and A. D. Auerbach, "Spectrum of sequence variations in the FANCA gene: an International Fanconi Anemia Registry (IFAR) study," Human Mutation, vol. 25, no. 2, pp. 142-149, 2005.

[17] A. J. Tipping, T. Pearson, N. V. Morgan et al., "Molecular and genealogical evidence for a founder effect in Fanconi 
anemia families of the Afrikaner population of South Africa," Proceedings of the National Academy of Sciences of the United States of America, vol. 98, no. 10, pp. 5734-5739, 2001.

[18] E. Callen, J. A. Casado, M. D. Tischkowitz et al., "A common founder mutation in FANCA underlies the world's highest prevalence of Fanconi anemia in Gypsy families from Spain," Blood, vol. 105, no. 5, pp. 1946-1949, 2005.

[19] A. P. Gillio, P. C. Verlander, S. D. Batish, P. F. Giampietro, and A. D. Auerbach, "Phenotypic consequences of mutations in the Fanconi anemia FAC gene: an international Fanconi anemia registry study," Blood, vol. 90, no. 1, pp. 105-110, 1997.

[20] M. Futaki, T. Yamashita, H. Yagasaki et al., "The IVS4 + 4A to T mutation of the Fanconi anemia gene FANCC is not associated with a severe phenotype in Japanese patients," Blood, vol. 95, no. 4, pp. 1493-1498, 2000.

[21] M. A. Whitney, H. Saito, P. M. Jakobs, R. A. Gibson, R. E. Moses, and M. Grompe, "A common mutation in the FACC gene causes Fanconi anaemia in Ashkenazi Jews," Nature Genetics, vol. 4, no. 2, pp. 202-205, 1993.

[22] D. Kutler and A. Auerbach, "Fanconi anemia in Ashkenazi Jews," Familial Cancer, vol. 3, no. 3-4, pp. 241-248, 2004.

[23] L. Faivre, P. Guardiola, C. Lewis et al., "Association of complementation group and mutation type with clinical outcome in Fanconi anemia," Blood, vol. 96, no. 13, pp. 40644070, 2000.

[24] T. Yamashita, N. Wu, G. Kupfer et al., "Clinical variability of Fanconi anemia (type C) results from expression of an amino terminal truncated Fanconi anemia complementation group C polypeptide with partial activity," Blood, vol. 87, no. 10, pp. 4424-4432, 1996.

[25] H. Joenje, "Fanconi anaemia complementation groups in Germany and the Netherlands," Human Genetics, vol. 97, no. 3, pp. 280-282, 1996.

[26] R. Kalb, K. Neveling, H. Hoehn et al., "Hypomorphic mutations in the gene encoding a key Fanconi anemia protein, FANCD2, sustain a significant group of FA-D2 patients with severe phenotype," American Journal of Human Genetics, vol. 80, no. 5, pp. 895-910, 2007.

[27] I. Demuth, M. Wlodarski, A. J. Tipping et al., "Spectrum of mutations in the Fanconi anaemia group $\mathrm{G}$ gene, FANCG/XRCC9," European Journal of Human Genetics, vol. 8, no. 11, pp. 861-868, 2000.

[28] A. D. Auerbach, J. Greenbaum, K. Pujara et al., "Spectrum of sequence variation in the FANCG gene: an International Fanconi Anemia Registry (IFAR) study," Human Mutation, vol. 21, no. 2, pp. 158-168, 2003.

[29] H. Yagasaki, T. Oda, D. Adachi et al., "Two common founder mutations of the fanconi anemia group $G$ gene FANCG/XRCC9 in the Japanese population," Human mutation, vol. 21, no. 5, p. 555, 2003.

[30] N. V. Morgan, F. Essop, I. Demuth et al., "A common Fanconi anemia mutation in black populations of sub-Saharan Africa," Blood, vol. 105, no. 9, pp. 3542-3544, 2005.

[31] J. Rosendorff and R. Bernstein, "Fanconi's anemiachromosome breakage studies in homozygotes and heterozygotes," Cancer Genetics and Cytogenetics, vol. 33, no. 2, pp. 175-183, 1988.

[32] B. P. Alter, "Diagnostic evaluation of FA," in Fanconi Anemia: Guidelines for Diagnosis and Management, M. E. Eiler et al., Ed., pp. 33-48, Fanconi Anemia Research Fund, Eugene, Ore, USA, 2008.

[33] M. Castella, R. Pujol, E. Callen et al., "Origin, functional role, and clinical impact of Fanconi anemia FANCA mutations," Blood, vol. 117, no. 14, pp. 3759-3769, 2011.
[34] N. Ameziane, A. Errami, F. Léveillé et al., "Genetic subtyping of Fanconi anemia by comprehensive mutation screening," Human Mutation, vol. 29, no. 1, pp. 159-166, 2008.

[35] J. R. Lo Ten Foe, M. T. Barel, P. Thuß, M. Digweed, F. Arwert, and H. Joenje, "Sequence variations in the Fanconi anaemia gene, FAC: pathogenicity of 1806insA and R548X and recognition of D195V as a polymorphic variant," Human Genetics, vol. 98, no. 5, pp. 522-523, 1996.

[36] H. Hanenberg, S. D. Batish, K. E. Pollok et al., "Phenotypic correction of primary Fanconi anemia T cells with retroviral vectors as a diagnostic tool," Experimental Hematology, vol. 30, no. 5, pp. 410-420, 2002.

[37] S. Chandra, O. Levran, I. Jurickova et al., "A rapid method for retrovirus-mediated identification of complementation groups in Fanconi anemia patients," Molecular Therapy, vol. 12, no. 5, pp. 976-984, 2005.

[38] T. N. Kaiser, A. Lojewski, C. Dougherty, L. Juergens, E. Sahar, and S. A. Latt, "Flow cytometric characterization of the response of Fanconi's anemia cells to mitomycin C treatment," Cytometry, vol. 2, no. 5, pp. 291-297, 1982.

[39] F. O. Pinto, T. Leblanc, D. Chamousset et al., "Diagnosis of Fanconi anemia in patients with bone marrow failure," Haematologica, vol. 94, no. 4, pp. 487-495, 2009.

[40] B. Hirsch, A. Shimamura, L. Moreau et al., "Association of biallelic BRCA2/FANCD1 mutations with spontaneous chromosomal instability and solid tumors of childhood," Blood, vol. 103, no. 7, pp. 2554-2559, 2004.

[41] J. E. Wagner, J. Tolar, O. Levran et al., "Germline mutations in BRCA2: shared genetic susceptibility to breast cancer, early onset leukemia, and Fanconi anemia," Blood, vol. 103, no. 8, pp. 3226-3229, 2004.

[42] K. Offit, O. Levran, B. Mullaney et al., "Shared genetic susceptibility to breast cancer, brain tumors, and Fanconi anemia," Journal of the National Cancer Institute, vol. 95, no. 20, pp. 1548-1551, 2003.

[43] S. Reid, D. Schindler, H. Hanenberg et al., "Biallelic mutations in PALB2 cause Fanconi anemia subtype FA-N and predispose to childhood cancer," Nature Genetics, vol. 39, no. 2, pp. 162 164, 2007.

[44] B. Xia, J. C. Dorsman, N. Ameziane et al., "Fanconi anemia is associated with a defect in the BRCA2 partner PALB2," Nature Genetics, vol. 39, no. 2, pp. 159-161, 2007.

[45] K. Somyajit, S. Subramanya, and G. Nagaraju, "RAD51C: a novel cancer susceptibility gene is linked to Fanconi anemia and breast cancer," Carcinogenesis, vol. 31, no. 12, pp. 20312038, 2010.

[46] J. P. Schouten, C. J. McElgunn, R. Waaijer, D. Zwijnenburg, F. Diepvens, and G. Pals, "Relative quantification of 40 nucleic acid sequences by multiplex ligation-dependent probe amplification," Nucleic acids research, vol. 30, no. 12, p. e57, 2002.

[47] A. R. Gennery, M. A. Slatter, A. Bhattacharya et al., "The clinical and biological overlap between Nijmegen Breakage syndrome and Fanconi anemia," Clinical Immunology, vol. 113, no. 2, pp. 214-219, 2004.

[48] P. van der Lelij, A. B. Oostra, M. A. Rooimans, H. Joenje, and J. P. de Winter, "Diagnostic overlap between Fanconi anemia and the cohesinopathies: roberts syndrome and Warsaw Breakage syndrome," Anemia, vol. 2010, Article ID 565268, 7 pages, 2010.

[49] J. R. Lo Ten Foe, M. L. Kwee, M. A. Rooimans et al., "Somatic mosaicism in Fanconi anemia: molecular basis and clinical significance," European Journal of Human Genetics, vol. 5, no. 3, pp. 137-148, 1997. 
[50] Q. Waisfisz, N. V. Morgan, M. Savino et al., "Spontaneous functional correction of homozygous Fanconi anaemia alleles reveals novel mechanistic basis for reverse mosaicism," Nature Genetics, vol. 22, no. 4, pp. 379-383, 1999.

[51] B. P. Alter, H. Joenje, A. B. Oostra, and G. Pals, "Fanconi anemia: adult head and neck cancer and hematopoietic mosaicism," Archives of Otolaryngology, vol. 131, no. 7, pp. 635-639, 2005.

[52] A. L. Medhurst, E. H. Laghmani, J. Steltenpool et al., "Evidence for subcomplexes in the Fanconi anemia pathway," Blood, vol. 108, no. 6, pp. 2072-2080, 2006.

[53] B. P. Alter, C. L. Frissora, D. S. Halperin et al., "Fanconi's anaemia and pregnancy," British Journal of Haematology, vol. 77, no. 3, pp. 410-418, 1991.

[54] P. S. Rosenberg, H. Tamary, and B. P. Alter, "How high are carrier frequencies of rare recessive syndromes? Contemporary estimates for Fanconi anemia in the United States and Israel," American Journal of Medical Genetics A, vol. 155, no. 8, pp. 1877-1883, 2011.

[55] A. D. Auerbach, "Fanconi anemia," in Diagnosis and Treatment of the Unborn Child, M. I. New, Ed., pp. 27-35, IdelsonGnocchi, Naples, Italy, 1999.

[56] A. Bechtold, R. Kalb, K. Neveling et al., "Prenatal diagnosis of Fanconi anemia: functional and molecular testing," in Fanconi Anemia. A Paradigmatic Disease for the Understanding of Cancer and Aging, D. Schindler and H. Hoehn, Eds., Monographs in Human Genetics, pp. 131-148, Karger, Basel, Switzerland, 2007.

[57] A. Merrill, L. Rosenblum-Vos, D. A. Driscoll, K. Daley, and K. Treat, "Prenatal diagnosis of Fanconi anemia (Group C) subsequent to abnormal sonographic findings," Prenatal Diagnosis, vol. 25, no. 1, pp. 20-22, 2005.

[58] D. I. Kutler, B. Singh, J. Satagopan et al., "A 20-year perspective on the International Fanconi Anemia Registry (IFAR)," Blood, vol. 101, no. 4, pp. 1249-1256, 2003.

[59] P. C. Verlander, A. Kaporis, Q. Liu, Q. Zhang, U. Seligsohn, and A. D. Auerbach, "Carrier frequency of the IVS4 + $4 \mathrm{~A} \longrightarrow$ T mutation of the Fanconi anemia gene FAC in the Ashkenazi Jewish population,” Blood, vol. 86, no. 11, pp. 4034-4038, 1995.

[60] M. Berwick, J. M. Satagopan, L. Ben-Porat et al., "Genetic heterogeneity among Fanconi anemia heterozygotes and risk of cancer," Cancer Research, vol. 67, no. 19, pp. 9591-9596, 2007.

[61] M. Tischkowitz, D. F. Easton, J. Ball, S. V. Hodgson, and C. G. Mathew, "Cancer incidence in relatives of British Fanconi anaemia patients," BMC Cancer, vol. 8, article 257, 2008.

[62] N. G. Howlett, T. Taniguchi, S. Olson et al., "Biallelic inactivation of BRCA2 in Fanconi anemia," Science, vol. 297, no. 5581, pp. 606-609, 2002.

[63] E. Levy-Lahad and E. Friedman, "Cancer risks among BRCA1 and BRCA2 mutation carriers," British Journal of Cancer, vol. 96, no. 1, pp. 11-15, 2007.

[64] N. Rahman, S. Seal, D. Thompson et al., "PALB2, which encodes a BRCA2-interacting protein, is a breast cancer susceptibility gene," Nature Genetics, vol. 39, no. 2, pp. 165167, 2007.

[65] M. J. Garcia, V. Fernandez, A. Osorio et al., "Analysis of FANCB and FANCN/PALB2 Fanconi anemia genes in BRCA1/2-negative Spanish breast cancer families," Breast Cancer Research and Treatment, vol. 113, no. 3, pp. 545-551, 2009.

[66] A. Meindl, H. Hellebrand, C. Wiek et al., "Germline mutations in breast and ovarian cancer pedigrees establish RAD51C as a human cancer susceptibility gene," Nature Genetics, vol. 42, no. 5, pp. 410-414, 2010.

[67] A. R. Meetei, M. Levitus, Y. Xue et al., "X-linked inheritance of Fanconi anemia complementation group B," Nature Genetics, vol. 36, no. 11, pp. 1219-1224, 2004. 


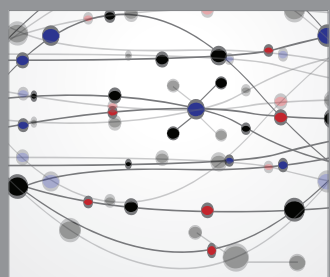

The Scientific World Journal
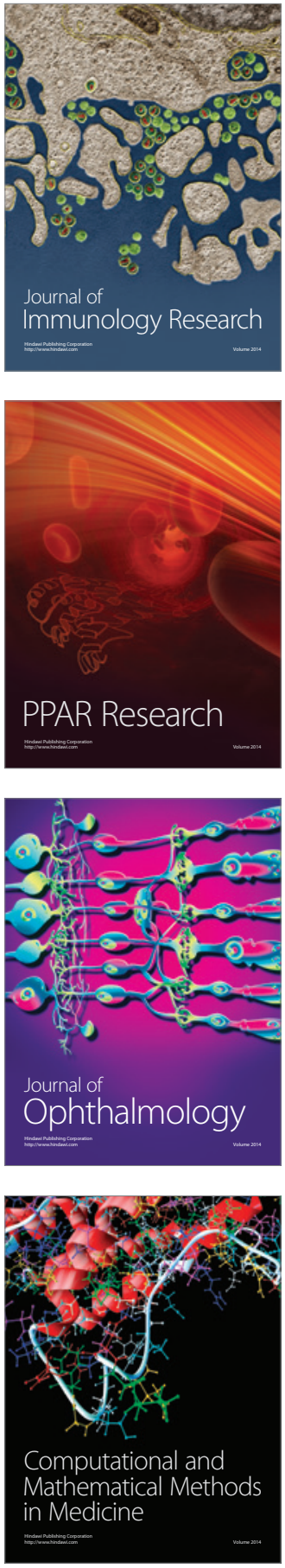

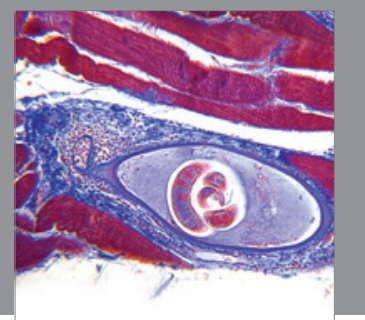

Gastroenterology

Research and Practice
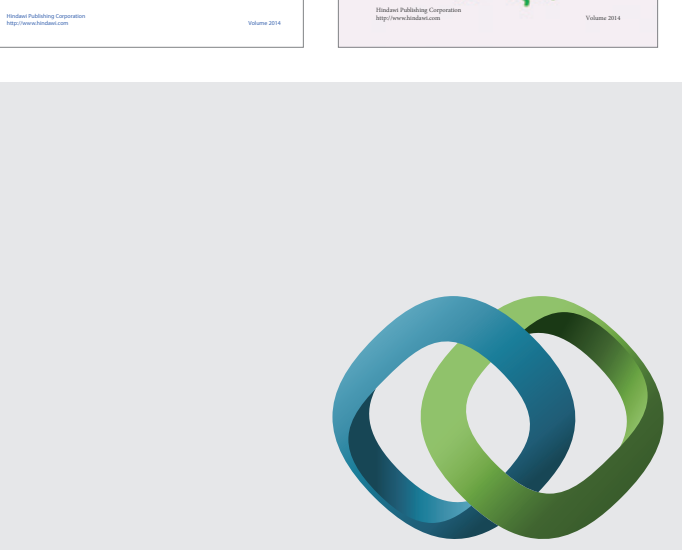

\section{Hindawi}

Submit your manuscripts at

http://www.hindawi.com
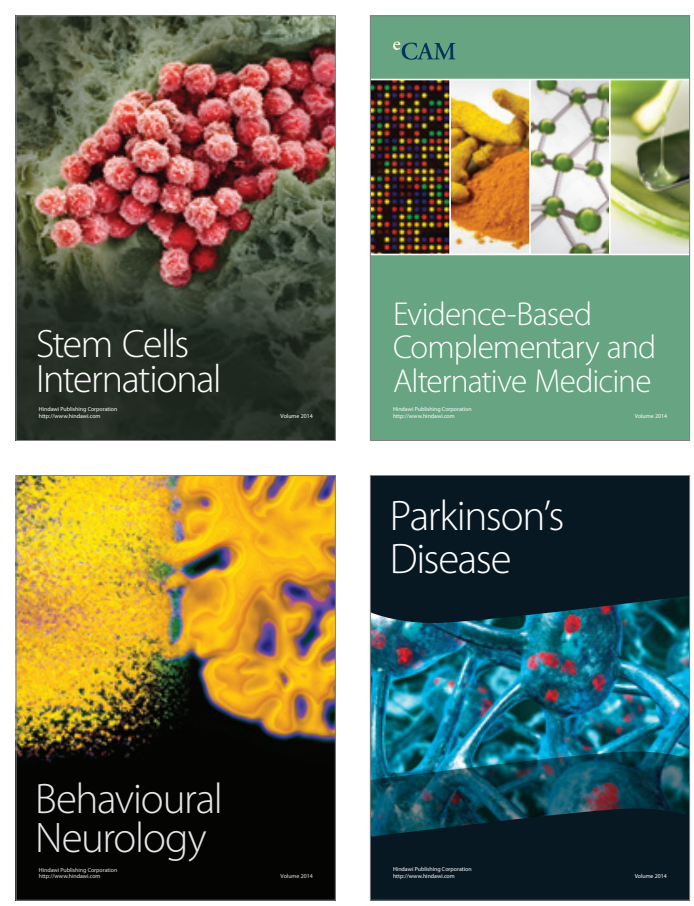

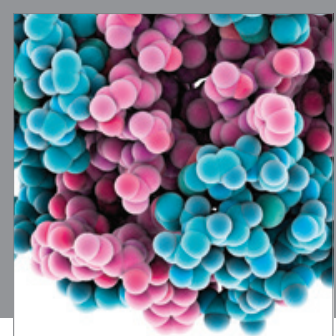

Journal of
Diabetes Research

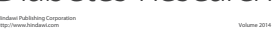

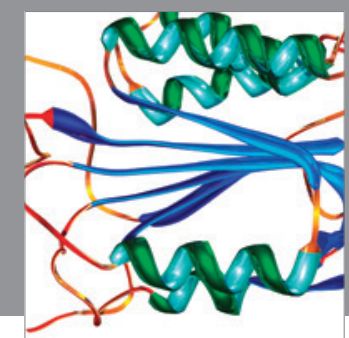

Disease Markers
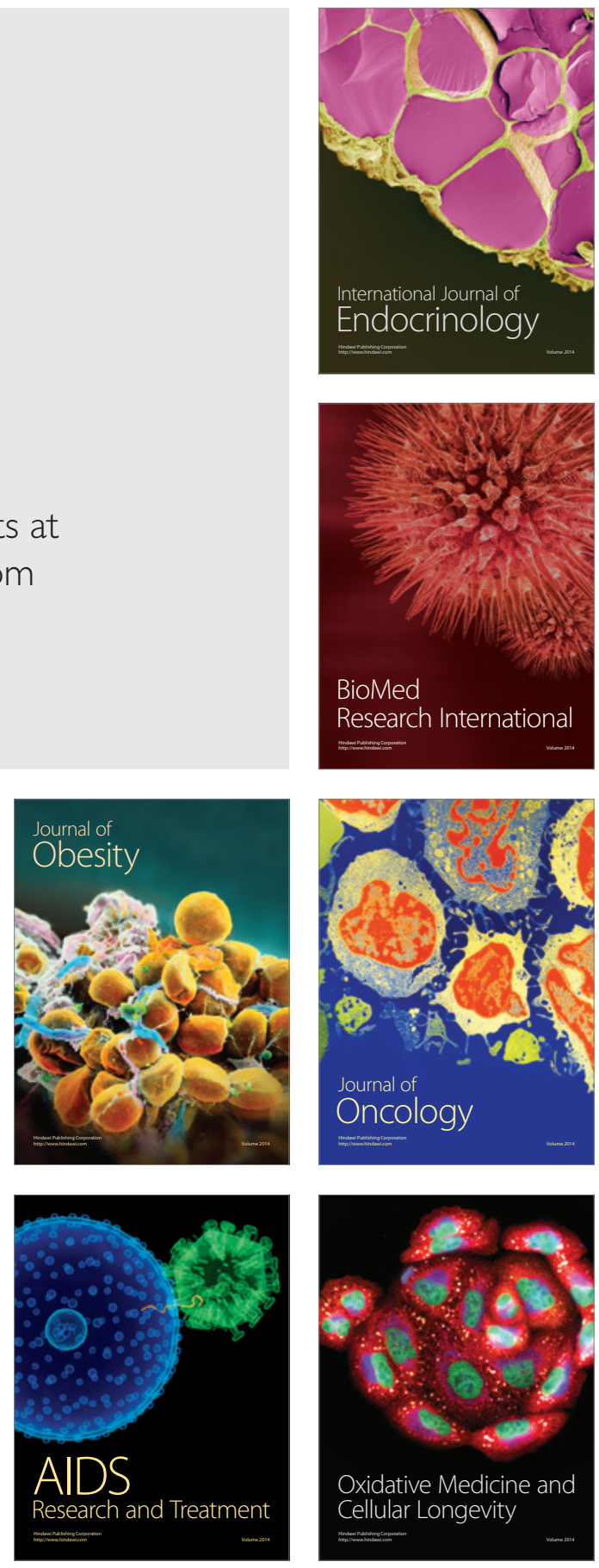